International Journal of Biomedicine I June 2019 - Volume 9, Issue Suppl_1: Abstracts From the Second Russian International Conference "Cryo-electron microscopy 2019: achievements and prospects"

POSTER ABSTRACT PRESENTATIONS

SESSION TITLE: STRUCTURE AND FUNCTIONS OF THE TRANSCRIPTION AND TRANSLATION APPARATUS OF THE CELL

DOI: 10.21103/IJBM.9.Suppl_1.P15

\title{
Abstract P-15: Structural Insights of Dirithromycin Binding to the Bacterial Ribosome
}

Evgeny Pichkur ${ }^{1,2,5}$, Pavel Kasatsky ${ }^{2}$, Alena Paleskava, ${ }^{2,3}$, Alexander Myasnikov ${ }^{2,6,7}$, Yury Polikanov ${ }^{8}$, Ilya A. Osterman ${ }^{4,9}$, Andrey Konevega ${ }^{1,2,3}$

${ }^{1}$ NRC "Kurchatov Institute", Moscow, Russia; ${ }^{2}$ NRC "Kurchatov Institute” - PNPI, Gatchina, Russia; ${ }^{3}$ Peter the Great St.-Petersburg Polytechnic University, St. Petersburg, Russia; ${ }^{4}$ Lomonosov Moscow State University, Department of Chemistry and A.N. Belozersky Institute of Physico-Chemical Biology, Moscow, Russia; ${ }^{5}$ FSRC "Crystallography and Photonics", Moscow, Russia; 'St. Jude Children's Research Hospital, Memphis, US; ${ }^{7}$ Centre for Integrative Biology (CBI), IGBMC, CNRS, Inserm, Université de Strasbourg, Illkirch, France; ${ }^{8}$ Department of Biological Sciences, University of Illinois, Chicago, USA; ${ }^{9}$ Skolkovo Institute of Science and Technology, Skolkovo, Russia

Background: Macrolide antibiotics bind in the nascent peptide exit tunnel (NPET) of the bacterial ribosome near the peptidyl transferase center. One of the second-generation macrolides, dirithromycin (DIR), differs from the parent erythromycin (ERY) by the presence of a hydrophobic (2-methoxyethoxy)methyl side chain that has significantly increased the delivery of this antibiotic to tissues due to better lipophilicity. In this work, we present a cryo-EM structure of DIR bound to the functional complex of 70S ribosome from E. coli.

Methods: Ribosomal complexes containing deacylated tRNA ${ }^{\mathrm{fMet}}$ in the $\mathrm{P}$ site and fMet-PhetRNA $^{\text {Phe }}$ in the A site were incubated with $30 \mathrm{mkM}$ DIR for $10 \mathrm{~min}$ before application onto carbon coated grids (Quantifoil R 2/2) and freezing. Cryo-EM data was collected using cryo-TEM Titan Krios and processed using Warp, Relion 3.0 and CisTEM. Model building was performed in Phenix.real_space_refine and Coot.

Results: Preliminary analysis failed to reveal any significant differences in location of macrolactone ring of DIR (current work) and ERY (pdb: 4v7u). In both structures only one hydrogen bond is formed between antibiotic and residue A2058. However, in comparison to the crystal structure of the ribosomal complex with ERY, our cryo-EM map demonstrates additional rotation of A2062 of $23 \mathrm{~S}$ rRNA towards desosamine of DIR.

Conclusion: Structural peculiarities of DIR binding to the E. coli ribosome determined by highresolution cryo-EM overall coincide with the mode of ERY interaction with the E. coli ribosome revealed 
by X-ray crystallography. Our results suggest a conformational lability of the (2-methoxyethoxy)-methyl side-chain of DIR, which is directed towards tunnel cavity and doesn't form any additional contacts with its walls.

Key Words: ribosome • antibiotics • structure $\bullet$ cryo-EM

Sources of Funding: This work was supported by RSF \#17-14-01416. The authors acknowledge the support and the use of resources of the Resource Center for Probe and Electron Microscopy at the NRC "Kurchatov Institute".

International Journal of Biomedicine. 2019;9 Suppl 1: S23. doi: 10.21103/IJBM.9.Suppl_1.P15

(C)2019 International Medical Research and Development Corporation 